\title{
Emerging Smart Grid Communication Technology for Mitigating Power Distribution Network Problems
}

\author{
Aparna S. Telang ${ }^{1}$, Prashant P. Bedekar ${ }^{2}$, Ashish K. Duchakke ${ }^{1}$ \\ ${ }^{1}$ Department of Electrical Engineering, Sant Gadge Baba Amravati University, Amravati, India \\ ${ }^{2}$ Department of Electrical Engineering, Gondwana University, Chandrapur, India
}

\section{Email address:}

aparna_telang2002@yahoo.com (A. S. Telang)

\section{To cite this article:}

Aparna S. Telang, Prashant P. Bedekar, Ashish K. Duchakke. Emerging Smart Grid Communication Technology for Mitigating Power Distribution Network Problems. Machine Learning Research. Vol. 4, No. 1, 2019, pp. 1-6. doi: 10.11648/j.mlr.20190401.11

Received: March 22, 2019; Accepted: April 26, 2019; Published: May DD, 2019

\begin{abstract}
Communication network is an integral part of an intelligent based fully automated smart grid system. It plays an important role in the framework of the transition towards distribution side of the smart grid system. Power theft, Fault detection, Overloading etc. are some of the important issues on the power distribution networks. To address these issues, a novel Arduino based prototype model "Smart Electricity System" has been proposed in this paper. It includes global system for mobile communication (GSM) for its effective implementation on the distribution network. Moreover another novel feature, Advanced Metering Infrastructure (AMI) is added to the proposed model. This is the key technology deployed on the distribution side of the smart grid system. The Uniqueness of the proposed model lies in the detection of power theft, where the information is sent to MSEB directly via interactive model of GSM 800 and APR voice kit, in the fault detection and its isolation by proper coordination between relay and Aurdino and in the overloading warning. Doing so, not only electricity is conserved but also the safety of living beings and protection of electrical appliances can be achieved effectively. Modern controllers with effective sensors are used to achieve all these issues for greater accuracy.
\end{abstract}

Keywords: Smart Grid, Communication Technology, Advanced Metering Infrastructure (AMI), Power Theft, APR Voice Kit, GSM

\section{Introduction}

Today's electric power distribution network is complex and stressed to meet the needs of $21^{\text {st }}$ century. Power theft, fault detection, overloading etc. are certain issues which make the distribution power sector unreliable and unsecure, a new grid infrastructure, smart grid has been emerged to address these issues. To exhibit the envisioned features of power grid into reality, smart grid integrates modern sensing technologies, control methodologies and advanced communications. These technologies improve the efficiency, reliability, economics and sustainability of the generation, transmission and distribution of electricity [1-2].

Communication is a major enabling technology for the smart grid [3]. Smart grid communication helps in improving power quality, fostering innovations to enable new products and services. Both wired and wireless communication technologies should be considered for the last mile communication access in the smart grid [4]. A comprehensive review on the recent works on the applications of wireless communicationtechnologies in the smart grid have been presented in [5]. The Advanced Metering Infrastructure (AMI) has been recognized as a key communication mechanism in the modern power distribution network [6-8]. It offers a two way communication network connecting millions of smart meters and sensors to utility centers. A through literature survey ofintelligent agents (intelligent entities) and smart grid applications of agents in the smart gridhas beendone in [9]. A description of communication requirements, potential applications on the SG roadmap has been presented in [10-11]. Reference [12] develops a system that assists in the detection of fault location with high accuracy and can monitor faults in real time, connecting the system responsible for managing the electric power grid through a telecommunications network. The concept of a high speed wireless network capable of real time monitoring and control of both loads and distributed generation across an isolated power system have been well presented in [13]. 
Reference [14] has discussed various SG security threats and challenges as well as giving a classification which could be refined and constantly updated to give a better model for addressing the security and resiliency issues right from the source of threats. Smart grid (SG) is emerging as a new facet of power industry. It incorporates numerous advanced technologies to deal issues prevailing with conventional electric networks. Considering the vision and roadmap of the Indian SG, an indicative assessment framework has been developed and details are discussed in [15]. Transmission and sub transmission lines are tapped to supply loads or integrate RRs such as wind or solar farms as medium-sized generation technologies. Modern protection schemes based on standalone decisions are not suitable for protecting a multitapped system with RRs at the transmission and sub transmission levels. A key element for future energy supply is ICT using IoE (Internet of Energy). Authors of [16] proposes a novel scheme referred to as the iprotectionmethod, which is one of the essential factors in producing energyreliably and efficiently by enhancing the process of system protectionusing information technology.

In this paper, a prototype model that allows investigation of certain major issues such as power theft, fault detection and overloading has been proposed. Moreover, automatic billing feature has been added to this model, for good monitoring and billing of electricity consumption. The proposed model consists of digital energy meter, an Arduino (micro-controller), GSM module, APR33A3 eight channel voice kit and current sensors. Thus altogether this smart electricity model tries to improve efficiency, reliability and security of the modern power distribution system.

\section{Smart Grid and Communication Technologies}

The smart grid is an electric power grid that attempts to intelligently respond to all the components with which it is interconnected, including suppliers and consumers, in order to deliver electric power services efficiently, reliably, economically, and sustainably [1].

Power Grid, Intelligent Systems and Communication Systems are the three basic blocks of smart grid. Figure 1 gives an idea of smart grid technology.

It integrates the different areas as Distributed Generation (DG), Advanced Metering Infrastructure (AMI), Distribution Automation (DA), Substation Automation (SA), Flexible Alternating Current Devices (FACTS) and Demand Response (DR). AMI is the key technology being widely deployed on the smart grid. It provides two way communications between customer and the utility. The smart grid introduces communication capability into various components of the powersystem to facilitate information exchange. The communication system is a "backbone" of the entire smart grid $[1,7,10]$. To meet the major smart grid communication requirements such as security, reliability, robustness, scalability and quality of service, both wired and wireless communication technologies are extensively used. As suggested in [10], power line communication, ZigBee, WLAN, cellular technologies or the internet are few examples of these technologies. Now a day's cellular technologies such as GSM, GPRS, 2G, 3G, 4G and WiMAX are extensively used [11]. For the work presented here, GSM technology has been used. GSM is a mobile communication modem. It stands for Global System for Mobile communication. It requires a SIM (Subscriber Identity Module) card to activate communication with the network.

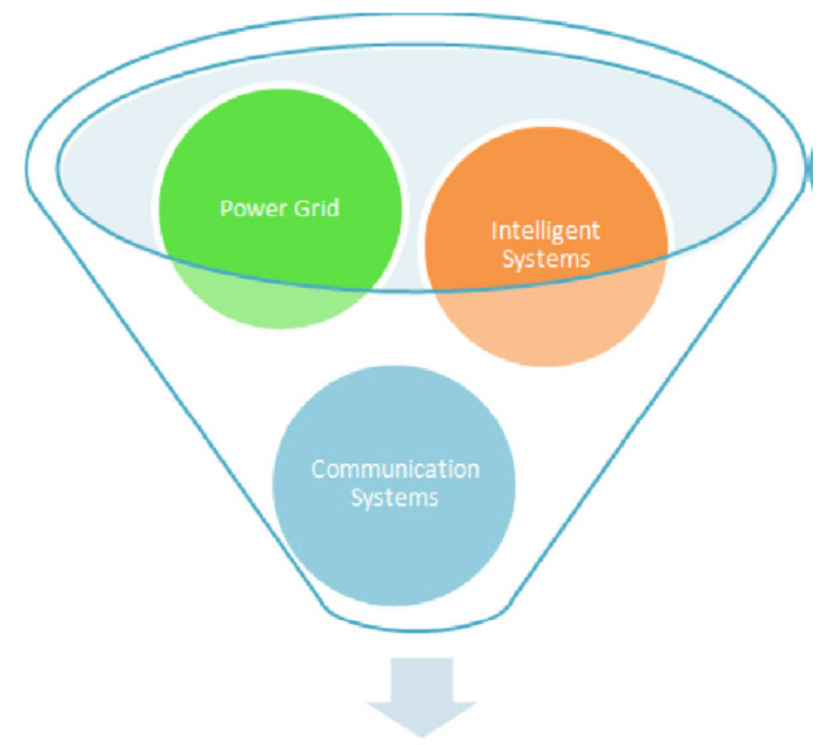

\section{Smart Grid}

Figure 1. Smart Grid basic blocks [9].

\section{Proposed Smart Electricity System Model}

The prototype model has been designed for the Indian distribution power sector context, specifically for rural areas. The attributes of this model are-

1) To sense the fault on transmission line and isolate the faulty section so as to save human being as well as wild animals from endanger situation.

2) To detect power theft that occurs on transmission line by illicit way or any other means.

3) To provide overload warning when value of current reaches to its high value with the help of Arduino and thus protecting the electrical appliances.

4) To provide automatic billing by simply counting the pulses of energy meter.

It consists of four modules to achieve the mentioned attributes. The power theft detection module consists of two current sensors along with GSM technology for spontaneous messaging to the concerned higher authority and with APR module for alarm sound. Over loading module deals with the over loading priority which turns off the load automatically, as per priorities settings of the consumer. This results in saving of electricity for domestic purpose. Another module deals with the automatic billing of household meter which 
sends energy units consumption via message to the respective user. The last module is for fault detection. The hardware that this model needs, is a basic Arduino kit that has been programmed accordingly the objectives like-power theft, fault detection, overloading and automatic billing. GSM technology has been extensively used for greater reliability of the proposed model.

\section{System Architecture}

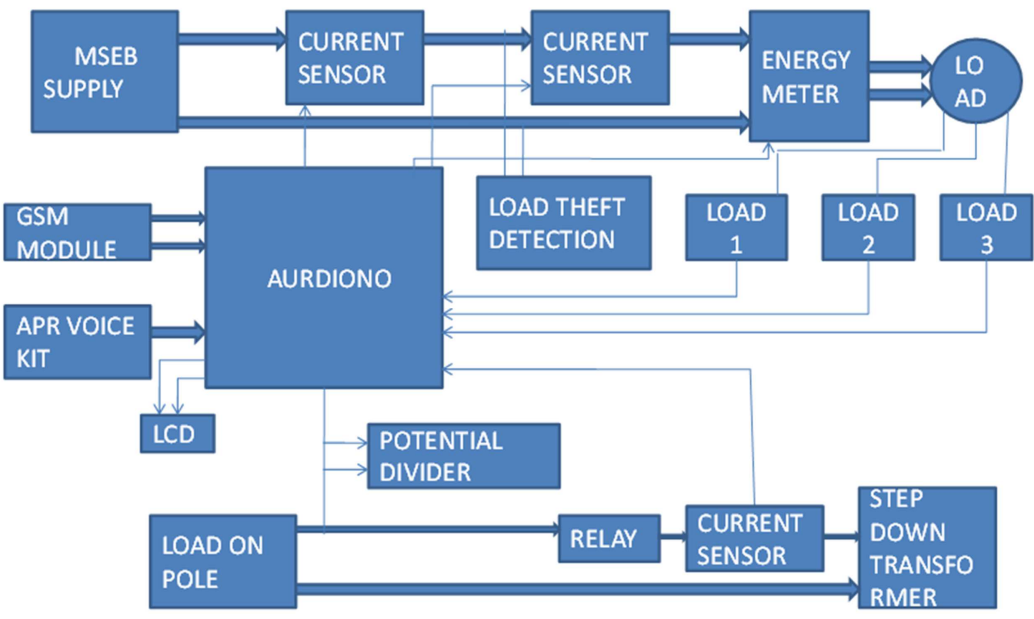

Figure 2. Block Diagram of Smart Electricity System.

It consists of digital energy meter, an Arduino (microcontroller), GSM module, APR33A3 eight channel voice kit, current sensors, relays, and step down transformer and LCD display.

\subsection{Arduino}

The core of the work presented here is Arduino board. It is a microcontroller and is open source hardware and software architecture shown in figure 3. It can control the smart devices connected to it such as sensors, GSM, Bluetooth, etc. Arduino is a preprogrammed device with boot loader that lets the user to upload the programs as per application requirement, into the chip flash memory.

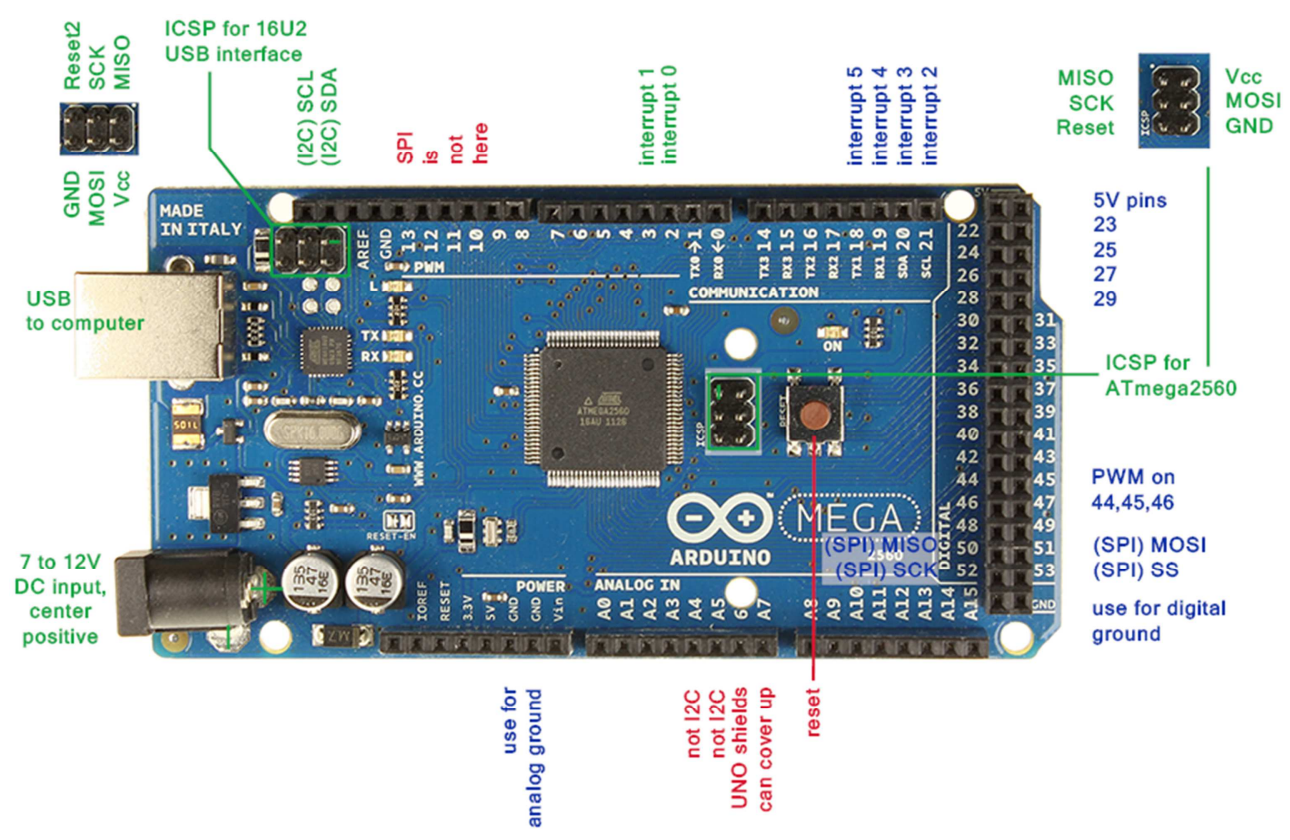

Figure 3. Arduino Board.

\subsection{GSM Modem}

GSM Modem has the capability to send and receive SMS to and from the system. The RS232 serial port is used for communication with the system. It needs AT commands for communicating with the microcontroller. This GSM Modem can accept any GSM network operator SIM card and act just like a mobile phone with its own unique phone number. GSM 
technology provides the benefit that the system is accessible in remote areas.

\subsection{APR Voice Kit}

Figure 4 shows 8 channel APR33A3 voice recorder and playback kit. The working voltage is 7 to $12 \mathrm{~V} \mathrm{AC} / \mathrm{DC}$ on power supply connector. Its recording time on all the 8 channels is 10 minutes.

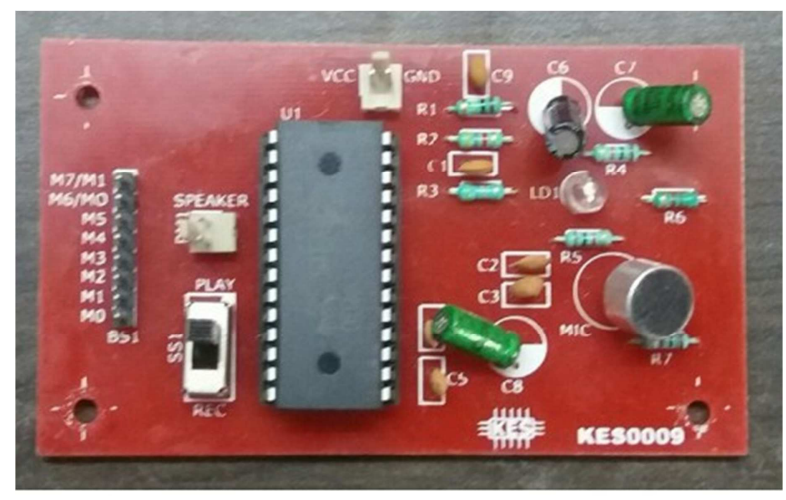

Figure 4. APR voice kit.

Interface the speaker pins and power on the module, slide the switch towards the recording option, then press and hold the M1 button and speak near the microphone. Release the button M1. To playback the recorder message, slide the switch towards playback and then just click the M1 button, the recorder message will be played. Repeat the same procedure to record the different messages on the different channels from M1 to M8.

\section{Results and Discussions}

Figure 5 shows hardware implementation of the proposed prototype model of smart electricity system. The model has been developed to achieve all objectives such as Power theft, Fault detection, over loading and automatic billing.

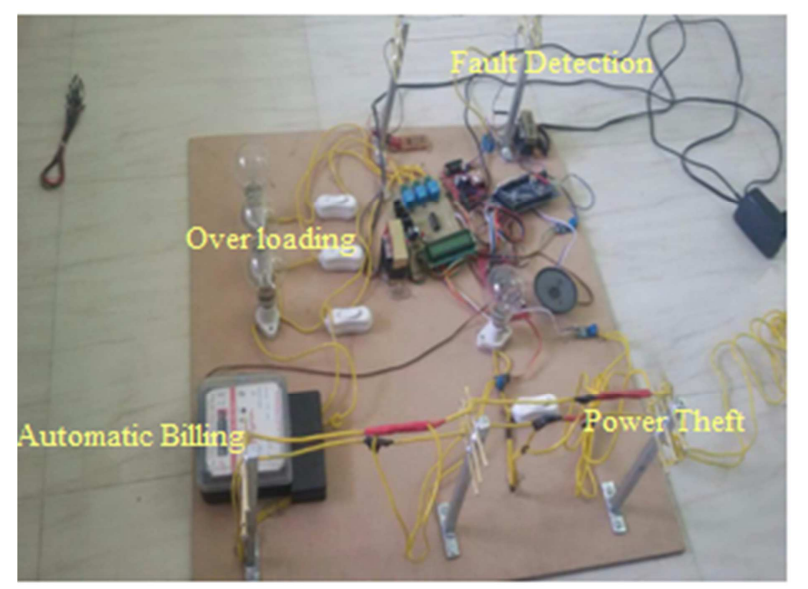

Figure 5. System Implementation Circuit diagram.

Case I. Power Theft Detection.

Power theft is a huge problem when serving power to a consumer. Different types of power theft are Outright Theft, which is accomplished by tappingan overhead line to create a new, illegal connection, Induction Coupling, Fraud accomplished by Bypassinga meter to prevent it from measuring the power consumed, Tamperingwith a meter, and concerned with Billing Issues.

In the present work, the current sensors connected at two different points for power theft detection. These current sensor senses the incoming and outgoing current and if any difference occurs then the Ardiuno senses thedifferential current value. APR module coupled with the speaker gets activated and gives the warning buzz "theft detected". At the same instant, GSM module sends SMS to the MSEB authority, so that the action against power theft would be taken immediately. The display on LCD connected in the model is as shown in figure 6 .

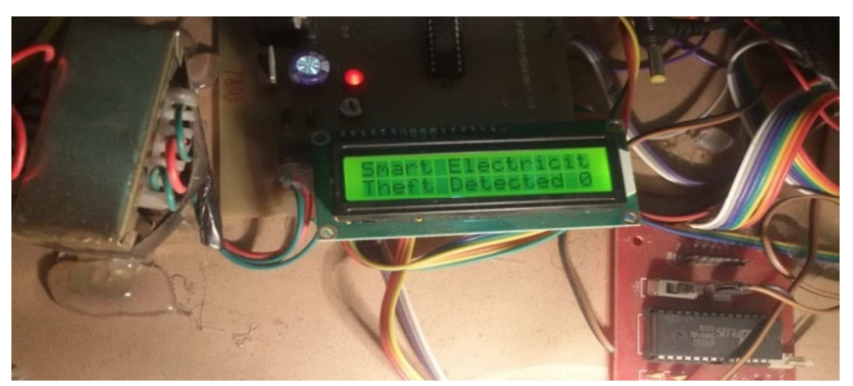

Figure 6. Power theft detection.

Case II. Fault Detection.

The different types of the electrical faults are L-G fault, LL-G fault, L-L fault, three phase fault. The most common and dangerous fault that occurs in a power system is the short circuit or shunt fault. When short circuit fault occur on the line, the value of fault current increases to a high extent and voltage drops. This faulty condition is sensed by the Arduino. As fault occur on the line, value of current suddenly increases and will be sensed by current sensors connected to the Arduino. Arduino will provide the delay of fraction of seconds and command the relay to trip the circuit and isolate the faulty section. So also the fault occurrence information will be sent to the MSEB, so that the fault can be cleared as early as possible. In prototype model, LCD will provide the display on LCD screen as "line faultoccur" as shown in figure 7.

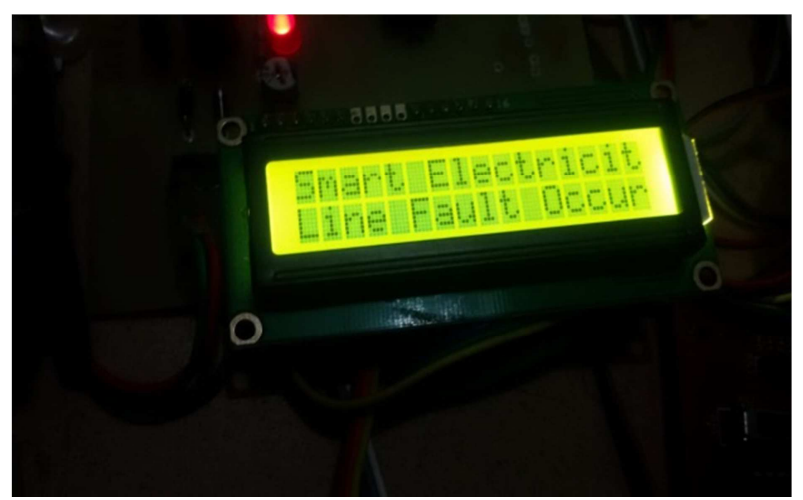

Figure 7. Fault detection. 
$80-90 \%$ of faults on any overhead line network are transient in nature. The remaining $10 \%-20 \%$ of faults are either semi-permanent or permanent. Transient faults are commonly caused by lightning and temporary contact with foreign objects. The immediate tripping of one or more circuit breakers clears the fault. Subsequent re-energization of the line is usually successful. A small tree branch falling on the line could cause a semi-permanent fault. The cause of the fault would not be removed by the immediate tripping of the circuit, but could be burnt away during a time-delayed trip. HV overhead lines in forest areas are prone to this type of fault. Permanent faults, such as broken conductors, and faults on underground cable sections, must be located and repaired before the supply can be restored. Use of an autoreclose scheme to re-energize the line after a fault trip permits successful re-energization of the line. Sufficient time must be allowed after tripping for the fault arc to de-energize prior to reclosing otherwise the arc will restrike. Such schemes have been the cause of a substantial improvement in continuity of supply. A typical single-shot auto-reclose scheme can be used.

Case III. Overload Protection.

When the overload occurs on any system, the value of current reaches to the high value which may damage the equipments. During overload, the current exceeds the prior set value, so Arduino can detect the overload condition and activate the APR module which is giving the warning "overload, please switch of the load" three times as set into the Arduino program. Moreover, there might be possibility of automatic switching off the load after alarming warning. The LCD display is shown in figure 8 .

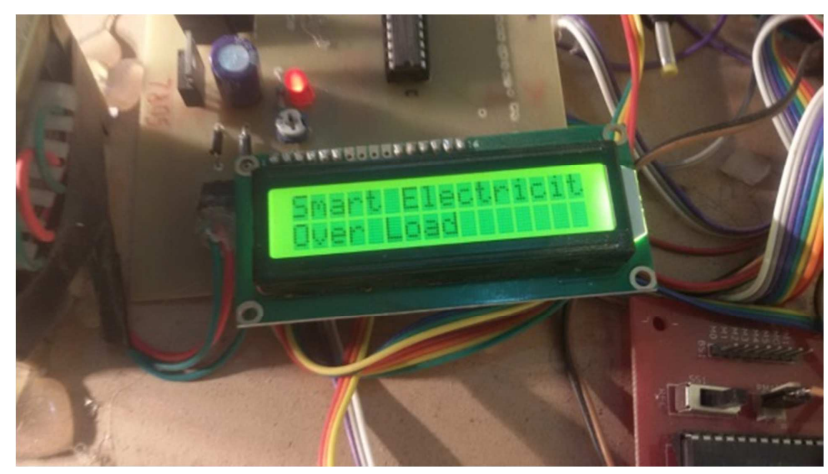

Figure 8. Overload protection.

Case IV. Automatic Billing.

Usually for the consumption of electricity, tariff billing system is usually adopted in general. Hence it becomes necessary to know, how much and where the actual consumption of electricity is taking place. For this purpose, Automatic billing feature of AMI technologyhave been incorporated in this model. Here simply the pulses of consumed electricity have been counted and then these pulses are fed back to Arduino. When the billing switch is pressed, the total consumed pulses are counted by Arduino and sent to the consumer via message in the environment of GSM module (3200 pulses). Figure 9 shows LCD display of automatic billing system.

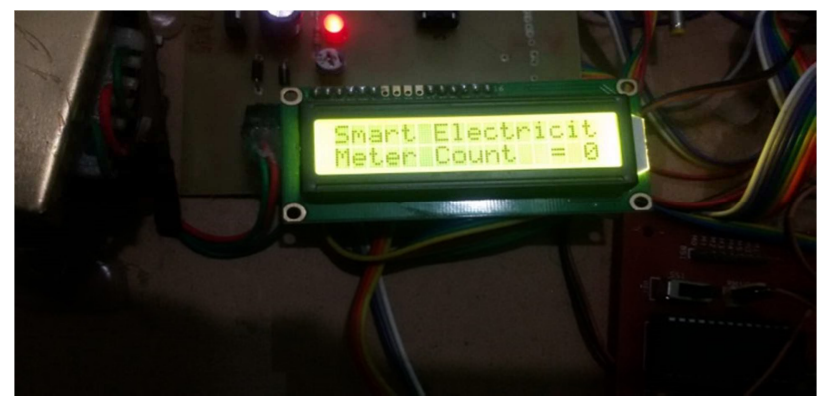

Figure 9. Automatic Billing.

\section{Conclusions}

Arduino based prototype model proposed in this paper addresses major issues like power theft, overloading protection, fault detection to achieve reliable and secure operation of smart power distribution network. A smart grid communication technology such as GSM has been used for managing information flow between utility and the consumer effectively. The addition of automatic billing feature of AMI to this model enhances the system performance in terms of conservation energy.

To make the proposed model more global in a present digital scenario, IOT sensors as well as GPS technology can be adopted in the system as a future scope. For practical applications Arduino used in the proposed model can be replaced by PLC-SCADA interface. Also more smart meters and other sensors such as phasor measurement units (PMU) can be deployed in the system. It is this smart electricity system that will work seamlessly in an existing Indian power distribution sector, particularly in rural areas.

Thus managing the power grid efficiently in terms of automatic billing feature of AMI and identifying power theft, protecting from faults and overloading are the most important features of the proposed model. This makes the smart power distribution network reliable, secure, flexible and scalable.

\section{Acknowledgements}

This work is supported by Vrushali P. Pere, Ritesh D. Bhudke, Anushree R. Dhule, Kiran S. Patil, Madhav S. Gangane, Kiran G. Bhele

\section{References}

[1] Vehbi C. Gungor, DilanSahin, TaskinKocak, SalihErgut, ConcettinaBuccella, Carlo Cecati, Gerhard P. Hancke, " Smart Grid Technologies: Communication Technologies and Standards," IEEE Transactions on Industrial Informatics, vol. 7, no. 4, pp. 529-538, November 2011.

[2] Jing Jiang and Yi Qian, "Distributed Communication Architecture for Smart Grid Applications," IEEE Communications Magazine, pp. 60-67, December 2016. 
[3] Stephen F. Bush, Sanjay Goel, Georges Simard, "IEEE Vision for Smart GridCommunications: 2030 and Beyond Roadmap," IEEE smart grid research association, pp. 1-19, 2013.

[4] Jingfang Huang, Honggang Wang, Yi Qian, "Smart Grid Communications in ChallengingEnvironments," IEEE smart grid symposium, pp. 552-557, 2012.

[5] Ruofei Ma, Hsiao-Hwa Chen, Yu-Ren Huang, and WeixiaoMeng, "Smart Grid Communication: Its Challenges and Opportunities," IEEE Transactions on Smart Grid, pp. 110, October 2012.

[6] Z. Lia, F. Yanga, S. Mohagheghib, Z. Wanga, J. C. Tourniera, Y. Wang"TowardSmart DistributionManagement by Integrating Advanced Metering Infrastructure," Electric Power Systems Research 105 (2013) 51- 56, July 2017.

[7] Saurabh Amin, Galina A. Schwartz, Alvaro A. CÁrdenas, and S. Shankar Sastry, "Game-Theoretic Models of Electricity Theft Detection in Smart Utility Networks: Providing New Capabilities with Advanced Metering Infrastructure,"IEEE Transaction on Control Systems, vol. 35, Issue 1, pp. 66-81, Feb. 2015.

[8] Rong Jiang, Rongxing Lu, Ye Wang, Jun Luo, ChangxiangShen, and Xuemin (Sherman) Shen, " EnergyTheft Detection Issues for Advanced Metering Infrastructure in Smart Grid," Tsinghua Science And Technology, vol. 19, no. 2, pp. 105-120, April 2014.

[9] Farhan H. Malik, MattiLehtonenAalto, "A review: Agents in Smart Grids," Electric Power Systems Research, vol. 131, pp. 71-79, October 2015.

[10] V. Cagri Gungor, Dilan Sahin, Taskin Kocak, SalihErgut, Concettina Buccella, Carlo Cecati, and Gerhard P. Hancke, "Survey on Smart Grid Potential Applications
andCommunication Requirements," IEEE Transactions oOn Industrial Informatics, vol. 9, no. 1, pp. 28-42, February 2013.

[11] D. Baimel and S. Tapuchi, N. Baimel, "Smart Grid Communication Technologies-Overview, Research Challenges and Opportunities," International Symposium on Power Electronics, Electrical Drives, Automation and Motion, pp. 116-120, December2016.

[12] Eduardo F. Ferreira, J. Dionísio Barros, "Faults Monitoring System in the Electric Power Grid of Medium Voltage," The 8th International Conference on Sustainable Energy Information Technology (SEIT 2018), Procedia Computer Science, pp. 696-703, 2018.

[13] DusanNikolic, Michael Negnewhitsky, "Smart Grid in Isolated Power Systems - Practical Operational Experiences," Applied Energy Symposium and Forum, Renewable Energy Integration with Mini/Microgrids, Energy Procedia, vol. 159, pp. 466-471, 2019.

[14] AbdulrahamanOkinoOtuozea, MohdWazir Mustafa, Raja MasoodLarik, "Review-Smart grids security challenges: Classification by sources of threats," Journal of Electrical Systems and Information Technology, vol. 5, pp. 468-483, 2018.

[15] Ramakrishna Kappagantu, S. Arul Daniel, "Review Challenges and issues of smart grid implementation:A case of Indian scenario," Journal of Electrical Systems and Information Technology, vol. 5, pp. 453-467, 2018.

[16] M. M. Eissa, Medhat H. A. Awadalla, "Centralized protection scheme for smart grid integrated with multiple renewable resources using Internet of Energy," Global Transitions (science direct), vol. 1, pp. 50-60, 2019. 